\title{
Speckle Data in the New Bright Star Catalogue
}

\author{
WAYNE H. WARREN, JR. \\ Hughes STX Corp., NASA Goddard Space Flight Center, \\ Greenbelt, MD 20771, USA \\ DORRIT HOFFLEIT \\ Department of Astronomy, Yale University, \\ Box 6666, New Haven CT, 06511, USA
}

\begin{abstract}
The new fifth edition of the Bright Star Catalogue (BSC5) will include speckle interferometric data in specially allocated columns of the main catalog. Speckle observations have already been included for some 1,800 "bright" stars and more are expected from recent sources. In addition to the separations for resolved pairs, upper limits will be included for stars that have not been resolved. This paper describes how the speckle data have been incorporated into the catalog and gives examples of the new format.
\end{abstract}

\section{INTRODUCTION}

Over the last 15 years or so, speckle interferometric techniques have provided a large body of new data for double and multiple stars, particularly the brighter ones that are included in the Bright Star Catalogue. Although information from speckle observations was included in the remarks section of the fourth edition of the BSC (Hoffleit 1982), the multitude of more recent observations has resulted in our decision to incorporate speckle observations into the catalog proper. This decision was also prompted by the goal of the CHARA group (McAlister 1992, private communication) to observe all BSC stars using speckle interferometry and by the work of the U.S. Naval Observatory speckle program (Worley 1991, private communication), which focuses on visual double stars of somewhat wider separation. Thus, in addition to the data on visual double and multiple systems now contained in the main catalog and its remarks, specific columns for speckle data will be allocated in the fifth edition of the BSC.

\section{PRECEPTS AND PROCEDURE}

\subsection{Precepts}

The following guidelines have been used to incorporate speckle data into the new catalog:

1. When data from visual observations are present in the double-star columns ( $\Delta \mathrm{m}$, separation, number of components) representing double and multiple codes other than "S" (duplicity discovered by speckle interferometry), independent speckle data do not replace them, but are present as additional information. For code " $\mathrm{S}$ ", the separations are always from speckle observations, of course. 
2. When a star is unresolved by speckle interferometry, an upper limit and its epoch of observation are reported. For resolved pairs, the speckle separation and its associated epoch are reported.

3. When multiple observations (either resolved or unresolved) are available, the most recent known measure and its epoch are given.

4. The data in the speckle interferometry fields generally replace speckle information given in the remarks of the fourth edition, which have been removed from the fifth edition. However, additional information is given in the remarks when necessary to clarify the nature of the double or multiple system.

5. The total number of components in a system has been increased to include a speckle companion if it is a new component discovered by speckle interferometry.

\subsection{Procedure}

The main source of speckle data has been the second edition of the CHARA compilation of interferometric measurements of binary stars (McAlister \& Hartkopf 1988). The catalog was first reviewed for all observations of stars identified by their HR numbers. The HR-HD-WDS Cross Index (Table 5) was then used to find bright stars identified only by ADS, DM, or WDS designations in the main part of the catalog. All data were placed into a separate file containing only HR number, measured separation (or upper limit), epoch of observation, and component identifiers (where necessary). This source file (ordered by HR number) was used in conjunction with the main catalog to prepare a list containing DM numbers and WDS identifiers (constructed from the J2000.0 equatorial coordinates included in the BSC5) to allow the identification of each star in the observational double-star database (WDSOBS) maintained at the U.S. Naval Observatory. This list contained only resolved systems, since measurements of unresolved stars are not included in the database. The WDSOBS was then examined for each system to check existing data and to look for observations more recent than the cutoff date (September 1988) of the CHARA catalog. The final speckle data file was then merged into the main BSC5 catalog.

\section{EXAMPLES}

The following entries show a few examples of how double-star data will appear in the new BSC5. Components will be identified separately for the speckle data if they are not identical to those included for visual data, or if there might be an ambiguity as to which component was observed. 


\begin{tabular}{|c|c|c|c|c|c|c|c|c|c|}
\hline HR & . & & Sep. & Epoch & $\begin{array}{l}\text { Speckle } \\
\text { Comp. }\end{array}$ & $\Delta \mathrm{m}$ & Sep. & $\begin{array}{l}\text { Visual } \\
\text { Comp. }\end{array}$ & $\mathrm{N}$ \\
\hline 1043 & . & & 0.325 & 1979.77 & $\mathrm{Aa}$ & 1.4 & 2.5 & $A \operatorname{axB}$ & 3 \\
\hline 6064 & . & . & $<0.038$ & 1985.52 & B & 1.0 & 6.4 & $\mathrm{AB}$ & 4 \\
\hline 6194 & . & & 0.212 & 1988.25 & $\mathrm{Ba}$ & 1.2 & 69.6 & $\mathrm{AxBa}$ & 4 \\
\hline 6623 & . & e & 1.621 & 1985.50 & Bc & 6.4 & 33.8 & $\mathrm{AxBc}$ & 4 \\
\hline 7048 & • & & 0.083 & 1990.27 & $\mathrm{Aa}$ & 0.4 & 2.7 & $\mathrm{~A} a \times \mathrm{B} B$ & 4 \\
\hline
\end{tabular}

\section{SUMMARY}

Speckle interferometric data have been incorporated into the new edition of the Bright Star Catalogue to complement the information on visual double and multiple systems already contained in the compilation.

\section{ACKNOWLEDGMENTS}

We thank H. A. McAlister and W. I. Hartkopf for speckle data received in advance of publication. We are indebted to C. E. Worley for many useful discussions and for allowing us to access the observational double-star catalog at the U. S. Naval Observatory.

\section{REFERENCES}

Hoffleit, D. (with the collaboration of Jaschek, C.) 1982, The Bright Star Catalogue, 4th revised edition (New Haven, Yale Univ. Observatory)

McAlister, H.A. \& Hartkopf, W.I. 1988, Second Catalog of Interferometric Measurements of Binary Stars, CHARA Contrib. No. 2 (Atlanta, Center for Higher Angular Resolution Astronomy, Georgia State Univ.) 